\title{
Erratum to: Recent Progress in JAK Inhibitors for the Treatment of Rheumatoid Arthritis
}

\author{
Shingo Nakayamada ${ }^{1} \cdot$ Satoshi Kubo $^{1} \cdot$ Shigeru Iwata $^{1} \cdot$ Yoshiya Tanaka $^{1}$
}

Published online: 3 October 2016

(c) Springer International Publishing Switzerland 2016

\section{Erratum to: BioDrugs \\ DOI 10.1007/s40259-016-0190-5}

Page 5, column 2, section 4.1, paragraph 2, line 25: The following dosage, which previously read " 3 or $5 \mathrm{mg}$ BID" should read "5 or $10 \mathrm{mg}$ BID".

Page 6, table 4, 'ORAL-standard phase III' study, 'Intervention' column:

2nd row, which previously read "MTX + Tofa 3 mg BID" should read "MTX + Tofa $5 \mathrm{mg}$ BID"

3rd row, which previously read "MTX + Tofa 5 mg BID" should read "MTX + Tofa $10 \mathrm{mg}$ BID"

The online version of the original article can be found under doi:10.1007/s40259-016-0190-5.

Yoshiya Tanaka

tanaka@med.uoeh-u.ac.jp

Shingo Nakayamada

s-nakaya@med.uoeh-u.ac.jp

1 The First Department of Internal Medicine, School of Medicine, University of Occupational and Environmental Health, 1-1 Iseigaoka, Yahata-nishi, Kitakyushu 807-8555, Japan 